\title{
Anesthetic Dilemma for Management of Parturient with Severe COVID-19 Pneumonia for Emergency Lower Segment Cesarean Section
}

\author{
Kewal K Gupta ${ }^{1}$, Gagan Deep ${ }^{2}$, Amanjot Singh ${ }^{3}$, Rupinder Kaur ${ }^{4}$, Himani Garg ${ }^{5}$
}

\begin{abstract}
Perioperative management of the pregnant patient with symptomatic coronavirus disease-2019 (COVID-19) presents a unique challenge to provide optimal medical care to both the mother and baby simultaneously along with protection of the healthcare providers from infection. Here, we report a unique case of a 28-year-old at 34 weeks of gestation with severe COVID-19 pneumonia posted for lower segment cesarean section which was successfully managed under spinal anesthesia. We aim to highlight the importance of neuraxial anesthesia in pregnant patients with symptomatic COVID-19 pneumonia.

Keywords: Cesarean section, Coronavirus disease 2019, Parturient, Perioperative management, Spinal anesthesia.

Research and Innovation in Anesthesia (2021): 10.5005/jp-journals-10049-0109
\end{abstract}

\section{INTRODUCTION}

Peripartum services are indispensable despite the coronavirus disease-2019 (COVID-19) pandemic. ${ }^{1}$ Providing safe and quality obstetric anesthesia is quite demanding especially in this second wave of COVID-19 where many pregnant patients are presenting with moderate to severe disease when compared with mostly asymptomatic cases in the first wave which has undoubtedly threatened the lives of mothers and neonates. In a recent study, $45 \%$ of the symptomatic COVID-19-positive pregnant women were presented with pneumonia and the requirement for intensive care unit (ICU) was $33.33 \%$ with a mortality rate of $11.11 \% .^{2}$ Till now, only a few cases of management in asymptomatic COVID-positive pregnant patients had been reported but here, we are presenting the first case of successful management of symptomatic COVID19 severe disease pneumonia in the pregnant patient which was posted for emergency lower segment cesarean section (LSCS).

\section{Case Description}

A 28-year-old female with 34 weeks of gestation was admitted to our tertiary care hospital because of COVID-19 pneumonia with cough and breathlessness. On examination, she was not in labor with the respiratory rate (RR) $26 \mathrm{~min}^{-1}$ and oxygen saturation $\left(\mathrm{SpO}_{2}\right) 86 \%$ on room air and $95 \%$ on oxygen by nasal prongs at 4 $\mathrm{L} \mathrm{min}^{-1}$. Any other medical complaint was ruled out except the past history of traveling 1 week before. As per COVID-19 protocol, all necessary investigations were sent and treatment including inj. dexamethasone with oxygen therapy started. Two days later, suddenly patient's condition worsened with $\mathrm{SpO}_{2}$ of $88 \%$ on a high-flow oxygen mask (HFOM) at $15 \mathrm{~L} \mathrm{~min}^{-1}$. So, in the interest of mother and child health, the patient was immediately planned for LSCS and shifted to COVID-19 designated operation theater (OT) which was equipped with negative pressure ventilation, anesthesia workstation, crash cart loaded with anesthetic and resuscitation drugs, and airway equipments including C-Mac video laryngoscope. On pre-anesthetic check-up, the patient was afebrile with NIBP of 130/86 mm Hg, HR of $98 \mathrm{~min}^{-1}$, RR of

\begin{abstract}
${ }^{1-5}$ Department of Anaesthesiology and Intensive Care, Guru Gobind Singh Medical College and Hospital, Faridkot, Punjab, India

Corresponding Author: Kewal K Gupta, Department of Anaesthesiology and Intensive Care, Guru Gobind Singh Medical College and Hospital, Faridkot, Punjab, India, Phone: +91-016399988316306, e-mail: doc_krishan31@yahoo.co.in

How to cite this article: Gupta KK, Deep G, Singh A, et al. Anesthetic Dilemma for Management of Parturient with Severe COVID-19 Pneumonia for Emergency Lower Segment Cesarean Section. Res Inno in Anesth 2021;6(2):51-53.
\end{abstract}

Source of support: Nil

Conflict of interest: None

$26 \mathrm{~min}^{-1}$ and $\mathrm{SpO}_{2}$ of $89 \%$ on HFOM. All the blood investigations including coagulation profile were within normal limits except total leukocyte count of $16,000 \mu \mathrm{L}^{-1}, \mathrm{C}$-reactive protein (CRP) of $145.3 \mathrm{mg} \mathrm{L}^{-1}$, and $D$ dimer level of $>10,000 \mathrm{ng} \mathrm{mL}^{-1}$. Chest $\mathrm{X}$-ray was showing bilateral consolidation but more on the right side (Fig. 1). We planned single-shot spinal anesthesia based on her general condition with a backup of non-invasive ventilation (NIV). The patient was explained about the procedure and written informed consent was taken. The entire team (anesthesiologists, obstetricians, neonatologists, nursing staff, and assistants) had used standard level 3 personal protective equipment (PPE) including N95 masks, jumpsuits, face shields, goggles, shoe covers, and double gloves. ${ }^{3,4}$ In OT, standard monitoring was applied to the patient and oxygenation continued with HFOM at a flow rate of $15 \mathrm{~L} \mathrm{~min}^{-1}$. Under all aseptic conditions, spinal anesthesia was given by a senior anesthesiologist in the left lateral position at the L3-L4 level with a $23 \mathrm{G}$ spinal needle and $2.0 \mathrm{~mL}$ (10 mg) of $0.5 \%$ heavy bupivacaine. After achieving the anesthetic level of T6, surgery started and a healthy female baby was delivered within 10 minutes of incision with APGAR score of 7 and 9 at 1 and 5 minutes, respectively, and was immediately transferred to neonatal isolation ward to minimize the risk of infection. The 


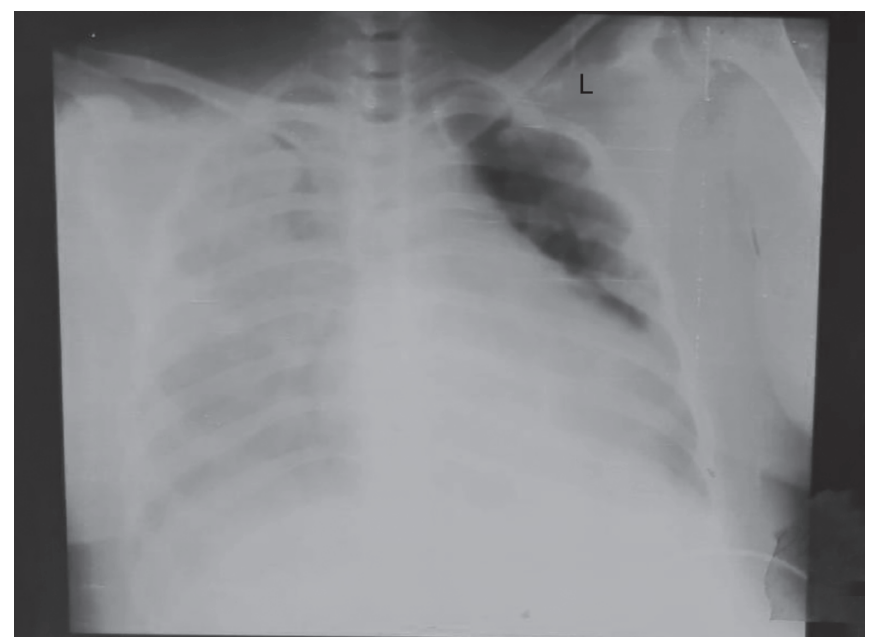

Fig. 1: Chest X-ray shows bilateral consolidation, more on the right side

patient's $\mathrm{SpO}_{2}$ improved immediately after delivery from 88 to $92 \%$ on HFOM, probably due to downward shifting of the diaphragm and improving lung compliance. Intraoperatively, the patient remained hemodynamically stable throughout the procedure and repeated counseling of the patient was done to allay her anxiety. Postoperatively, the patient was shifted to COVID ICU on HFOM for further management due to active COVID pneumonia and a few hours later, in view of desaturation along with tachypnea, the patient was put on NIV Bilevel support with $\mathrm{FiO}_{2}$ of $60 \%$. Standard COVID treatment protocol including inj. Remdesevir was given and $\mathrm{SpO}_{2}$ improved to $94 \%$. Due to a very high level of D-dimer, inj. Enoxaprine $0.6 \mathrm{~mL}$ subcutaneously started after 1 day of surgery. The patient was kept on NIV support in ICU for few days and the patient's clinical condition and chest X-ray started improving (Fig. 2), she was slowly weaned off from oxygen therapy to room air. After 10 days of hospital stay postoperatively, the mother was discharged home with a healthy baby as both were tested COVID negative.

\section{Discussion}

Healthcare workers are facing a new challenge every day as this COVID-19 virus of the second wave is more contagious and can cause life-endangering respiratory infections in mothers, neonates, and even healthcare professionals. Although an elective LSCS was considered to be the safest option, as the vaginal delivery after induction would require more exposure with more healthcare workers along with excessive aerosol production due to hyperventilation and risk of vertical transmission. ${ }^{5}$ But due to the paucity of well-accepted data regarding the timing and the safety of vaginal or cesarean delivery, the decision should be individually based on the maternal-fetal status, as well as obstetric indication. ${ }^{3,6}$ As in our case, the patient was getting hypoxic, so to prevent intrauterine fetal hypoxia and to improve pregnancy-induced physiologically compromised pulmonary compliance, LSCS was planned by an obstetrician.

In this case, after donning of PPE, a preanesthetic check-up was done in the preoperative area to minimize the delay in emergency surgery and to reduce the viral exposure. Although in some reported cases, pre-anesthetic check-ups through video calling had been done to avoid unnecessary COVID-19 exposure but it seems to be feasible only in elective cases and with stable patients. ${ }^{7}$

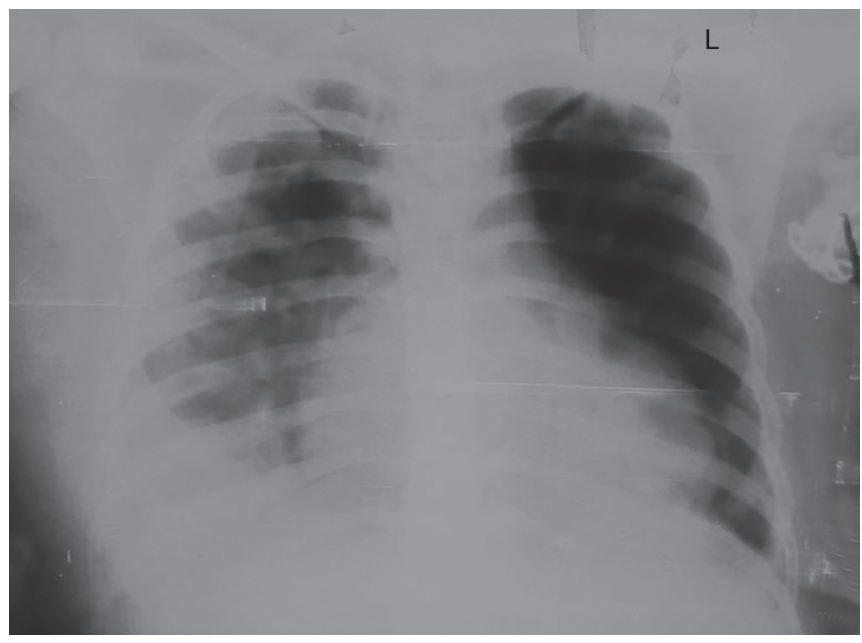

Fig. 2: Chest X-ray shows resolving consolidation

Assessing whether a parturient with COVID-19 is suitable for neuraxial procedures should focus on the risks of general anesthesia when compared with neuraxial anesthesia. General anesthesia should be considered when a COVID-19 parturient presents with desaturation $(\leq 93 \%)$ or for maternofetal reasons. ${ }^{8}$ However, recent reports have supported the safety of regional anesthesia for COVID-positive pregnant patients requiring LSCS, as anesthesiologists are at greatest risk of viral exposure during endotracheal intubations in these patients. Moreover, COVID-19 patients with hypoxia and concomitant physiologically decreased functional residual capacity from pregnancy will be likely to become more hypoxic, develop further atelectasis with intubation and mechanical ventilation, and possibly require postoperative critical care admission. ${ }^{3}$ In general, the risk of causing meningitis or encephalitis is extremely low with neuraxial procedures, even in infected patients. Hence, we planned rapid sequence spinal anesthesia to avoid risk and morbidity associated with the use of intubation and mechanical ventilation. In rapid sequence spinal anesthesia, pregnant patients are transferred in a left lateral position with supplemental oxygen, and a single shot subarachnoid block is provided by the most experienced, available anesthetist ${ }^{4}$ and the time required is comparable to general anesthesia with better neonatal outcomes. Both anesthesia and surgery should be performed by experts to ensure reduced exposure time. Although due to wearing of level 3 protection measures, difficulty to put spinal or epidural anesthesia has been reported in few cases $^{4,7}$ but with use of $23 \mathrm{G}$ spinal needle and experienced anesthesiologist, we achieved it comfortably in the first attempt. As this was the case of COVID-19 severe disease, postoperatively the patient was managed in ICU and recovered well.

We had used a fully equipped COVID-19 designated OT with a specific area for donning and doffing of PPE and having a separate exit facility for staff and positive patients as per Indian Society of Anaesthesiologist advisory. ${ }^{9}$

\section{ConClusion}

Spinal anesthesia can be used effectively and safely in COVID19-positive obstetric patients, especially in patients with active pneumonia, although more evidence are needed on the anesthetic care of these patients. All pregnant women with COVID-19 
pneumonia should be managed by multidisciplinary expert teams in a tertiary care hospital and all healthcare workers involved in the LSCS should properly use level 3 PPE equipment.

\section{ACKnOWLedgments}

We would like to thank Dr Nishi Garg and her team (from the Department of Obstetrics and Gynaecology) and all our colleagues especially Dr Seema Jindal (COVID ICU), Dr Arushi, and all staff of OT and ICU whose help and cooperation were invaluable in patient management and in preparing the manuscript.

\section{Consent of Patient}

The authors certify that they have obtained all appropriate patient consent forms.

\section{Contributions}

Kewal Krishan Gupta, Amanjot Singh and Gagan Deep: These authors helped in case management and manuscript writing and editing. Rupinder Kaur and Himai Garg: These authors helped in case management and manuscript editing. All authors read and approved the final version of the manuscript for publication.

\section{References}

1. Zhu N, Zhang D, Wang W, et al. A novel coronavirus from patients with pneumonia in China, 2019. N Engl J Med 2020;382(8):727-733. DOI: 10.1056/NEJMoa2001017.
2. Karasu D, Kilicarslan N, Ozgunay SE, et al. Our anesthesia experiences in COVID-19 positive patients delivering by cesarean section: a retrospective single-center cohort study. J Obstet Gynaecol Res, Wiley 2021. Available from: 10.1111/jog.14852.

3. Chen R, Zhang $Y$, Huang $L$, et al. Safety and efficacy of different anesthetic regimens for parturients with COVID-19 undergoing cesarean delivery: a case series of 17 patients. Can J Anaesth 2020;67(6):655-663. DOI: 10.1007/s12630-020-01630-7.

4. Chhabra A, Rao TN, Kumar M, et al. Anaesthetic management of a COVID-19 parturient for caesarean section - case report and lessons learnt. Indian J Anaesth 2020;64(Suppl 2):S141-S143. DOI: 10.4103/ ija.IJA 509_20.

5. Fornari F. Vertical transmission of Covid-19-a systematic review. J Pediatr Perinatol Child Health 2020;4(02):7-13. DOI: 10.26502/ jppch.74050034.

6. Parazzini F, Bortolus R, Mauri PA, et al. Delivery in pregnant women infected with SARS-CoV-2: a fast review. Int J Gynaecol Obstet 2020;150(1):41. DOI: 10.1002/ijgo.13166.

7. Malik A, Pallapotu B. Anesthetic management of a cesarean section in COVID-positive pregnant patient. Apollo Med 2020;17(0):224-225. DOI: 10.4103/am.am_61_20.

8. Ashokka B, Loh MH, Tan $\mathrm{CH}$, et al. Care of the pregnant woman with COVID-19 in labor and delivery: anesthesia, emergency cesarean delivery, differential diagnosis in the acutely ill parturient, care of the newborn, and protection of the healthcare personnel. Am J Obstet Gynecol 2020., Elsevier BV 2020;223(1):66-74.

9. Kinsella SM, Girgirah K, Scrutton MJ. Rapid sequence spinal anaesthesia for category-1 urgency caesarean section: a case series. Anaesthesia 2010;65(7):664-669. DOI: 10.1111/j.13652044.2010.06368.x. 